\title{
Paleopathology and osteobiography of the people of Peñuelas, Chile's semiarid north
}

\author{
Maria Araya Rosado ${ }^{+}$, Jessica Vernacchio-Wilson
}

Department of Geography and Anthropology, Rowan University, 201 Mullica Hill Rd., Glassboro, NJ 08028, US

The Museo de La Serena, IV Region, Chile has collections of skeletal remains representing the agricultural Diaguita people of 500 years ago excavated in the 1980s from the sites Peñuelas 21 and 24, Chile's semiarid north. Their excellent preservation has permitted an osteobiographical and radiographic analysis to better understand the patterns of the disease. This research continues the osteological analyses begun in 1989 by Rosado that seek to understand the impact the transition to and adoption of farming had on the health of prehistoric populations. Because of the significance of paleopathology in the understanding of cultural and biological adaptations, it has also become necessary to assess the preservation status and design a conservation protocol to protect and document the remains. The objectives of this communication are to: establish demographic patterns of the skeletal samples and identify and diagnose skeletal paleopathologies via photography and radiographs. Intentional cranial alteration, limb and cranial fractures, dental wear, and dental abscesses and caries are among the interesting paleopathologies so far documented. Intentional cranial alteration is very common and is manifested as tabular erect in both males and females. The high frequency of carious lesions indicates a diet that emphasized carbohydrates. Skeletal radiographs are available for several of the individuals in the sample and this has afforded a more detailed description of the paleopathologies originally documented via photography.

Key words: osteology - osteobiography - paleopathology - demography

Chile's semiarid north is well known for its excellently preserved osteological collections that covers a period of at least 3000 years and are elaborately documented through archaeology. Among these collections are those representing the Diaguita culture excavated in the early 1980s from Peñuelas (sector 21 and sector 24); a littoral site that has yielded human and animal skeletal remains dated to approximately 600 years ago. The sample represents a farming, pastoralist, and maritime people who settled Chile's semiarid's north over a thousand years ago (Biskupovic 1982, Ampuero 1989). The rather intact preservation of the remains has permitted an osteobiographical documentation and identification of paleopathological conditions which is available in a database housed at the Museo de La Serena. This database is currently used as baseline information to understand biological and cultural adaptations of prehistoric people of Chile's littoral.

Of much consideration to the archaeologists interested in this region is to understand the health patterns among prehistoric populations of the semiarid littoral and the impact on health resulting from farming subsistence activities. Paleopathology (Ortner \& Putschar 1885, Roberts \& Manchester 1999, Mays 2000) offers reasonably useful indicators about level of health by identifying diseases in the past, their demographic patterns, and frequencies among archaeological populations practicing the varying subsistence strategies. Paleopathology studies

${ }^{+}$Corresponding author: rosado@rowan.edu

Received 20 July 2006

Accepted 16 October 2006 are of continued benefit and needed in this region of Chile because of ongoing excavations (Biskupovic 1999), which yield more skeletal remains of different time periods; and because they facilitate a more complete reconstruction of life patterns and cultural adaptation, still largely unknown for the archaeological populations represented.

Because of the significant archaeological value in paleopathological investigations and the possibility afforded by the excellent preservation of the skeletal collections at the Museo de La Serena to document human diseases in the prehistoric past, it has also become necessary to examine the skeletal remains for preservation status, design a conservation protocol, and design a skeletal registry and database to better protect and document the remains respectively. This protocol has become an integral part of any physical anthropology investigation carried out at the Museo de La Serena.

The Peñuela's sample's excellent preservation can be largely attributed to the semiarid north's littoral climate that is temperate, or classified as Mediterranean. The annual rainfall is 1-6 inches and its mean annual temperature is $59^{\circ} \mathrm{F}$. Thus, the skeletal remains are buried in soils that do not experience alternating extreme levels of moisture and temperatures. In addition, the soil $\mathrm{pH}$ is neutral. These taphonomic conditions are conducive to preservation of organic remains, including human and animal bones.

The skeletal remains were excavated by Biskupovic (1982-1985) and Biskupovic and Ampuero (1989) from the littoral site of Peñuelas in Chile's Region IV which forms part of the semiarid north. The littoral is characterized by low coastal mountains and valleys and rivers that connect the high Andes to the coast, resulting in a very rugged topography. Contributing to this topography is the constant tectonic activity. It is not uncommon in the semiarid north for four or five years to pass without any pre- 
cipitation. The vegetation, therefore, is sparse and the valleys are populated by xeromorphic plant species that include many varieties of cacti.

The objectives of this study are to: (a) conduct an osteological analysis for identification and diagnosis of paleopathologies ; (b) expand the existing osteology and paleopathology database, and skeletal registry housed at the Museo de la Serena (uses the programs Lotus Approach and Microsoft Excel); (c) determine sex and age for demographic analysis; and d) measure long bones for stature determination.

\section{MATERIALS AND METHODS}

The osteological analysis comprises a skeletal blank that identifies the skeleton's site, sex, age, bones, number of bones per skeleton, and the state of preservation for each bone. The identification of state of preservation for each bone includes the categories: complete, incomplete, complete and broken, absent, and postmortem erosion. This also registers the side and number of bones present for each anatomical section, as well as descriptions of any anomalies and paleopathologies. A digital camera is used to photograph the bones and radiographs were also taken of bones with anomalies. With this system we are able to develop a bone- by- bone registry and a data base of anomalous conditions that include information on dental and skeletal paleopathologies, dental wear, occupational conditions, and artificial cranial deformation.

The age and sex of the individuals was ascertained by using gross osteological markers as described by Bass (1995) and Steele and Bramblett (1988). Characteristics used to determine subadult age include dental eruption, basilary suture, and epiphyseal union of long bones. Characteristics used to determine adult age include age related changes of the pubic symphysis, dental eruption of third molars, dental wear, epiphyseal union of long bones, union of sacral vertebras, osteoarthritic changes, and closure of cranial sutures. Anatomical characteristics of the pelvis and cranium, and in some cases specific measurements of bones, such as the glenoid fossa of the scapula and head of femur, were used to assign sex. The pelvis provided the most reliable traits for determining sex.

Stature was determined for adults with available complete femora. Although other long bones were available and were measured, the femora were used for the stature calculations. These provide more accurate stature determination according to Bass (1995). The formula used for the femur (in $\mathrm{cm}$ ) given by Bass for Mongoloid populations is: 2.15 femur $+72.57 \pm 3.80$.

\section{RESULTS}

Overall, the osteobiographical analysis of the sample indicate the individuals in the Peñuelas sample to be brachycephalic, of orthognathous faces with projecting zygomatics, wide noses, rounded chin, rounded dental arch, and shovel shaped incisors. These traits are consistent (Figs 1, 2, 3) with those found in peoples of Mongoloid ancestry, including the American Indians. The dental remains display moderate to severe dental wear, particularly of the premolars and molars.

The minimum number of individuals identified is 41

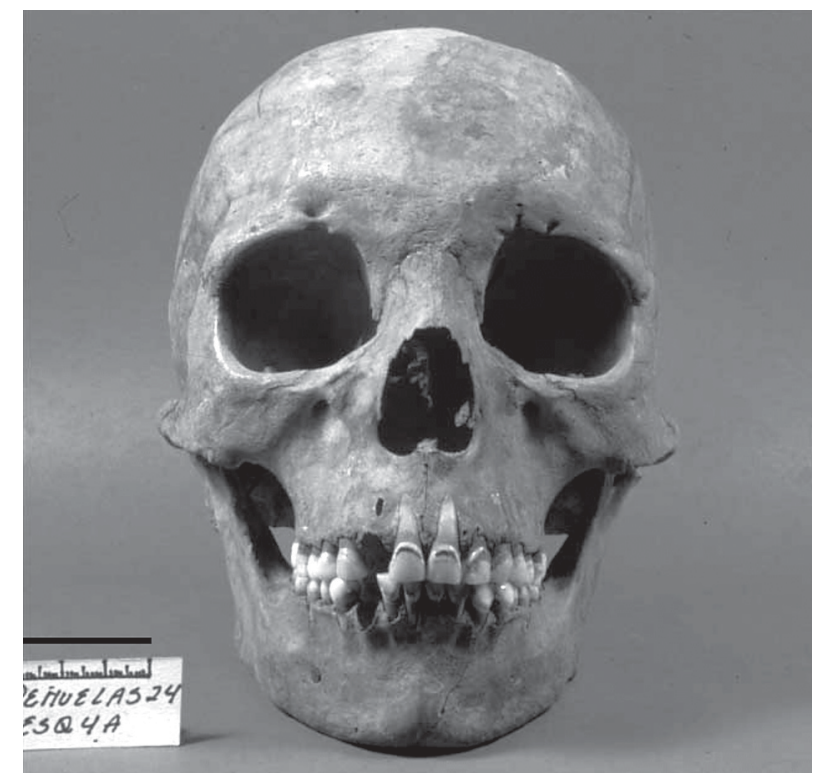

Fig. 1: frontal view, adult female, $45-50$ years. $B a r=3 \mathrm{~cm}$.

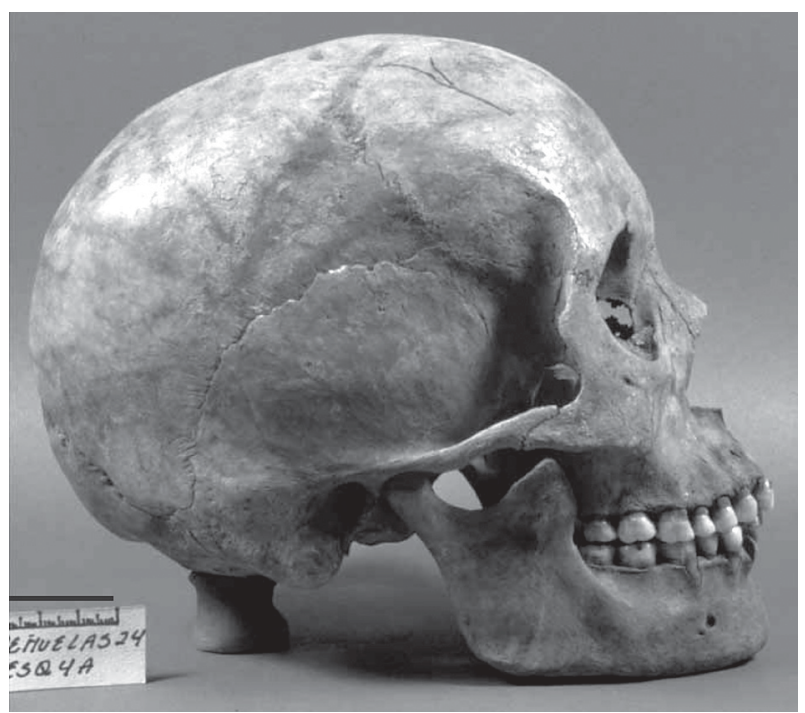

Fig. 2: sagittal view, adult female, $45-50$ years. $B a r=3 \mathrm{~cm}$.

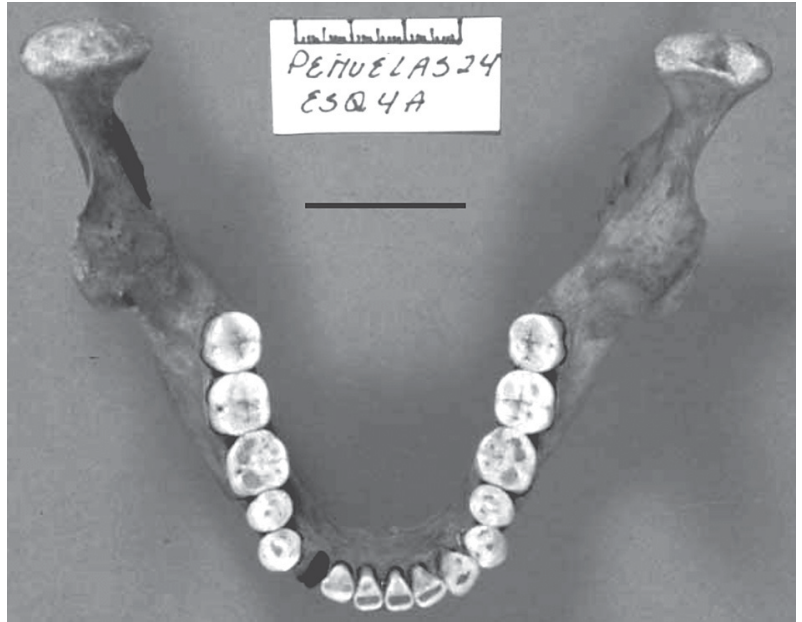

Fig. 3: superior view of mandible, adult female, 45-50 years. Bar $=$ $3 \mathrm{~cm}$. 
(Fig. 4), with several reaching their third, fourth, fifth, and sixth decades. The skeletal age ranges, as well as the sex, were ascertained with certainty because the various osteological markers for age and sex determination were available. Fig. 5 illustrates the minimum number of individuals distributed by sex (sample size by sex). Tables I and II illustrate the distribution of known and unknown sex. The unknown category includes the 11 subadults in the sample, therefore, $27 \%$ of this sample is not achieving adulthood. In Fig. 6 the age categories clearly show that, for the exception of two subadult individuals of unknown sex, the sample is made up of adults in their third through sixth decade. There were no infants represented in the sample. More females die in their third and fourth decades. These are the child bearing years and the higher frequency of age at death compared to males may be due to death during childbirth.

The male and female statures in Table III indicate sexual dimorphism in this respect. However, the differences between the sexes need to be tested for significance and on a larger sample. A future study on stature will generate stature estimation tables and formulas proper to populations of this region currently not available.

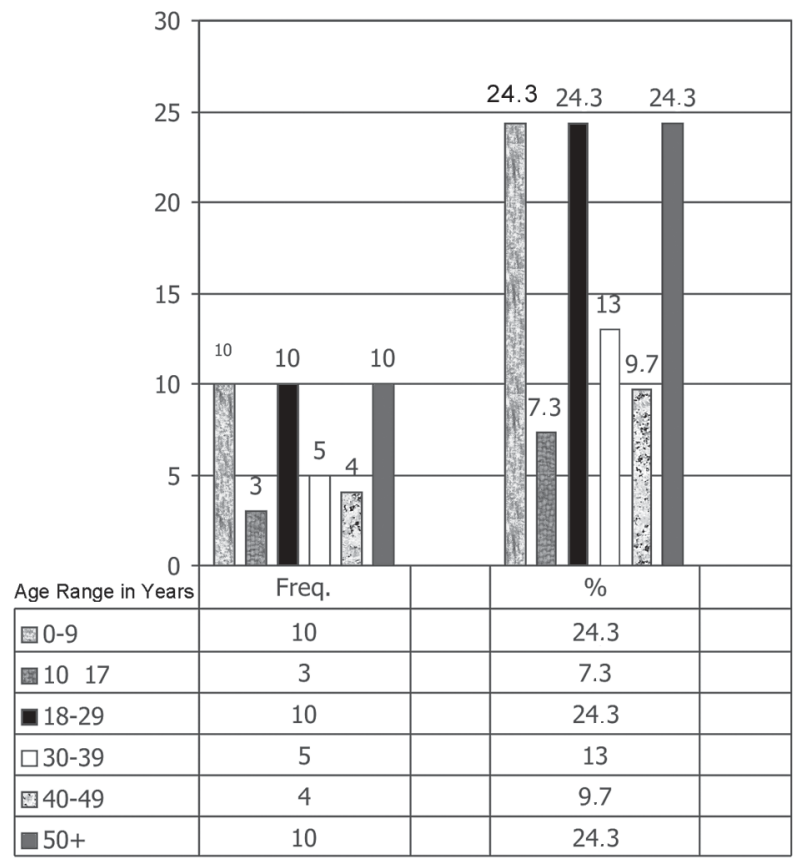

n (sample size): 41 (minimum number of individuals in the sample)

Fig. 4: age distribution.

TABLE I

Sample size by sex

\begin{tabular}{lcl}
\hline Sex & $\mathrm{n}$ (sample size) & $\%$ \\
\hline Male & 10 & 24.4 \\
Female & 16 & 39 \\
Unknown & 15 & 36.5 \\
\hline
\end{tabular}

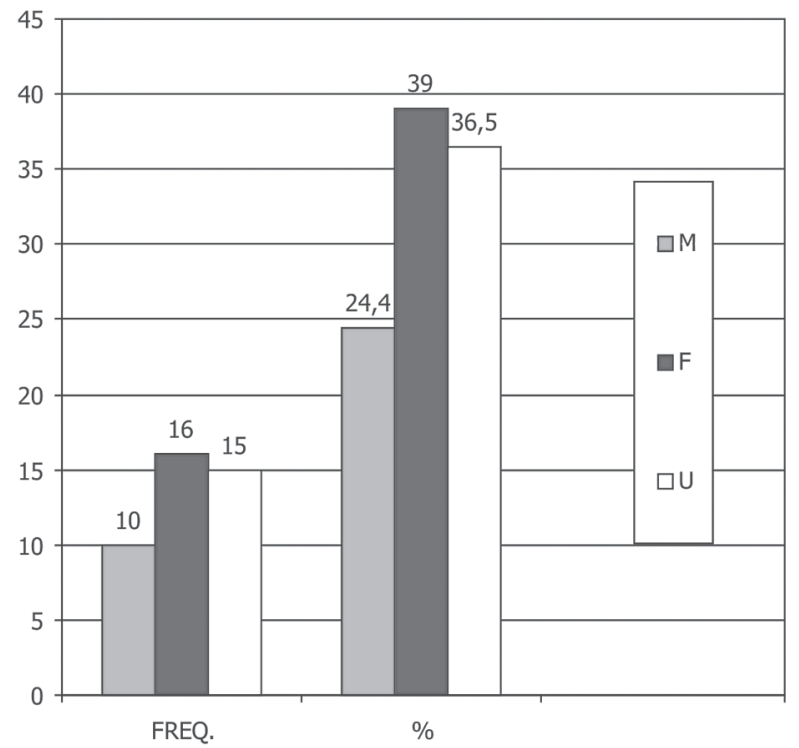

n: 41; M: male; F: female; U: unknown; n: sample size

Fig. 5: sample size by sex.

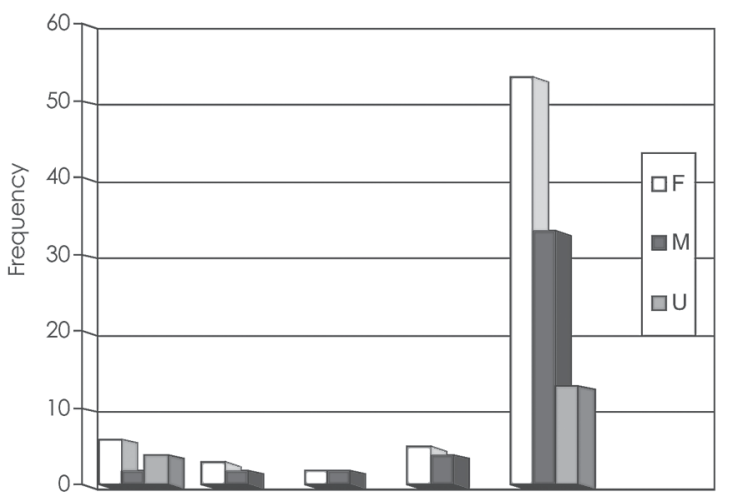

Age range in years

\begin{tabular}{|l|c|c|c|c|c|}
\cline { 2 - 6 } \multicolumn{1}{c|}{} & $18-29$ & $30-39$ & $40-49$ & $50+$ & $\%$ \\
\hline$\square F$ & 6 & 3 & 2 & 5 & 53 \\
\hline$\square M$ & 2 & 2 & 2 & 4 & 33 \\
\hline$\square U$ & 4 & & & & 13 \\
\hline
\end{tabular}

n (sample size): 30; sex not determinable for four adult individuals; F: female; M: male; U: unknown

Fig. 6: adult frequency of age by sex.

TABLE II

Distribution of unknown sex

\begin{tabular}{lc}
\hline Age range in yaers & $n$ unknown sex \\
\hline $0-9$ & 10 \\
$10-17$ & 1 \\
$18+$ & 4
\end{tabular}

$n$ : sample size.

To understand the impact of diet on the teeth the frequency of dental pathologies and degree of wear was determined. Caries, abscesses, periodontal disease, dental hypoplasia, antemortem tooth loss, and calculus formation were observed (Figs 9, 10, 11, 12). Fig. 7 illustrates the frequency of dental paleopathologies observed. There 
TABLE III

Adult stature

\begin{tabular}{cc}
\hline Adult Males (in cm) & Adult Females (in cm) \\
\hline-161.23 & -157.80 \\
-162.90 & -151.52 \\
-163.52 & -158.44 \\
-163.04 & -156.90 \\
-162.60 & \\
-165.30 & \\
Range: $161.23-165.30$ & Range: $151.52-158.44$
\end{tabular}

Formula used for the femur (in cm), from Bass (1995); Mongoloid: 2.15 femur $+72.57 \pm 3.80$; six male and four female with available paired femora were measured.

were 27 available dentitions of adults and subadults for analysis, each with paired maxillas and mandibles. The total affected by dental pathologies is 17 , or $63 \%$ of the sample. Two subadults, between 8 and 12 years of age, were affected by paleopathologies, but only caries; 17 adult individuals in the sample display clear cases of carious lesions. The lesions have destroyed the dentine and a few are clearly associated with abscess formation in the periapical region of the tooth affected by the caries. There are 9 individuals with abscesses. In several cases the abscess was severe enough that they penetrated the maxillary sinus. Carious lesions begin to appear by the second decade and abscesses by the third decade. The frequency of the dental paleopathologies observed is moderate to high and consistent with many published reports on the health status of sedentary, farming populations. (Cohen \& Armelagos 1984, Swedlund \& Armelagos 1990, Rosado 1998).

There are 29 mandibles and maxillas available for dental wear analysis (Fig. 8). The total number of individuals affected by dental wear is 21 and 20 are adults. Of the adults, 12 individuals display a severe degree of wear (grades 7 and 8 in Figs 10 and 12). In these cases the

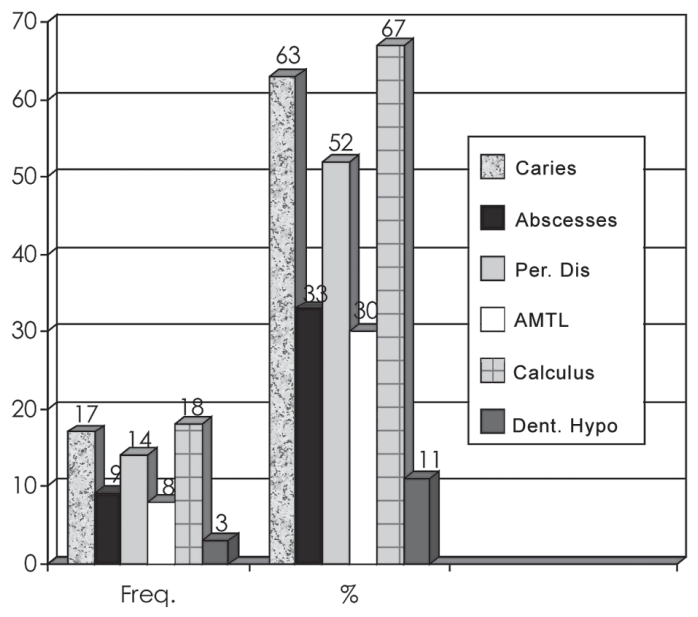

No. of individuals

$\mathrm{n}$ (sample of dentitions available): 27; Per.Dis: periodontal disease; AMTL: ante mortem tooth loss; Dent. Hypo: dental hypoplasia

Fig. 7: frequency of dental pathologies.

dentine is exposed and the patch is extensive, and the cusp pattern is obliterated; 8 individuals have a moderate form of dental wear. These individuals' crowns are somewhat obliterated and the dentine patch is minimal. The dental wear pattern for this sample is age dependent, with the molars and premolars being the most affected by severe wear starting in the second decade.

Cranial and postcranial paleopathologies-were identified. Table IV indicates the bone pathologies observed with the minimum number of individuals affected. Osteoarthritis, particularly of the vertebras, has the highest frequency, with the lumbar region being the most affected. Radiographs enable more accuracy in establishing correct diagnosis. Dr Morrie Kricun of the University of Pennsylvania, reviewed the radiographs taken for some of the individuals with clear markers of pathology. This helped us correlate the pathology markers evident in the radio-

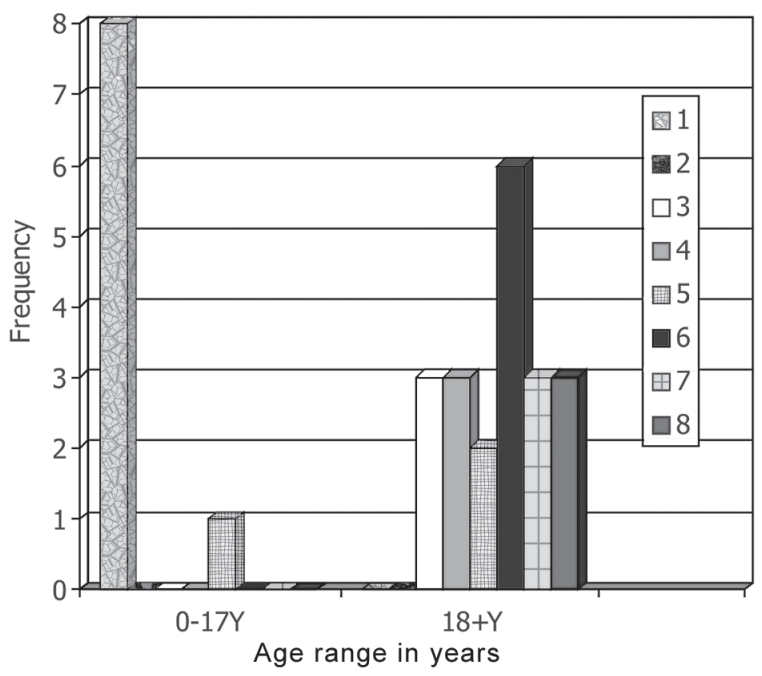

Wear Grade Categories, after Schmucker (1985)

1: unworn

2: wear facets minimal

3: cusp pattern obliterated

4: dentine patch, minimal

5: dentine patch, extensive

6: secondary dentine

7: crown (enamel) worn, root visible

8: roots functioning at occlusal level

Fig. 8: number of individuals affected by dental wear (grade category). 


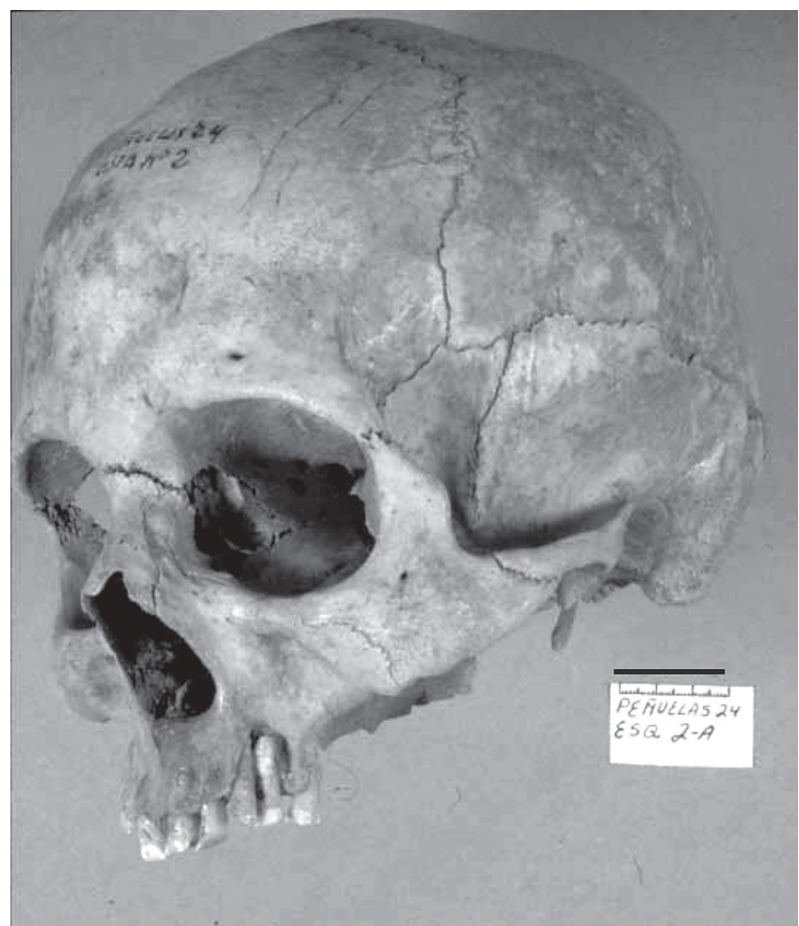

Fig. 9: abscess (left maxillary PM1). Bar: $3 \mathrm{~cm}$.

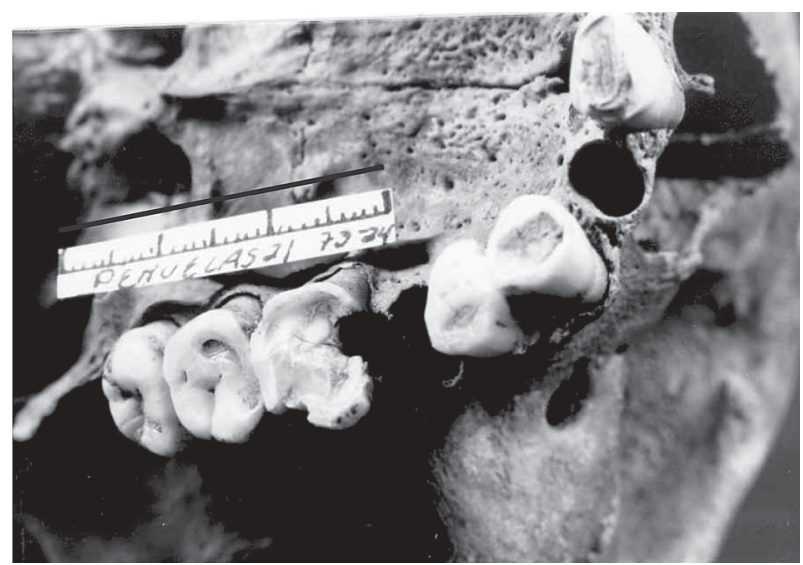

Fig. 10: caries and dental wear (right maxillary M1; wear grade 6; cusp pattern obliterated). Bar: $3 \mathrm{~cm}$.

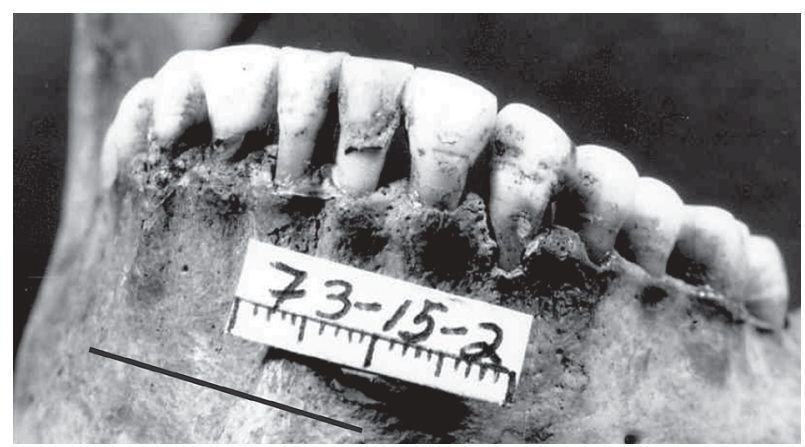

Fig. 11: periodontal disease (incisors), calculus (incisors, canine), and dental hypoplasia (lateral incisor, canine). Bar: $2 \mathrm{~cm}$.

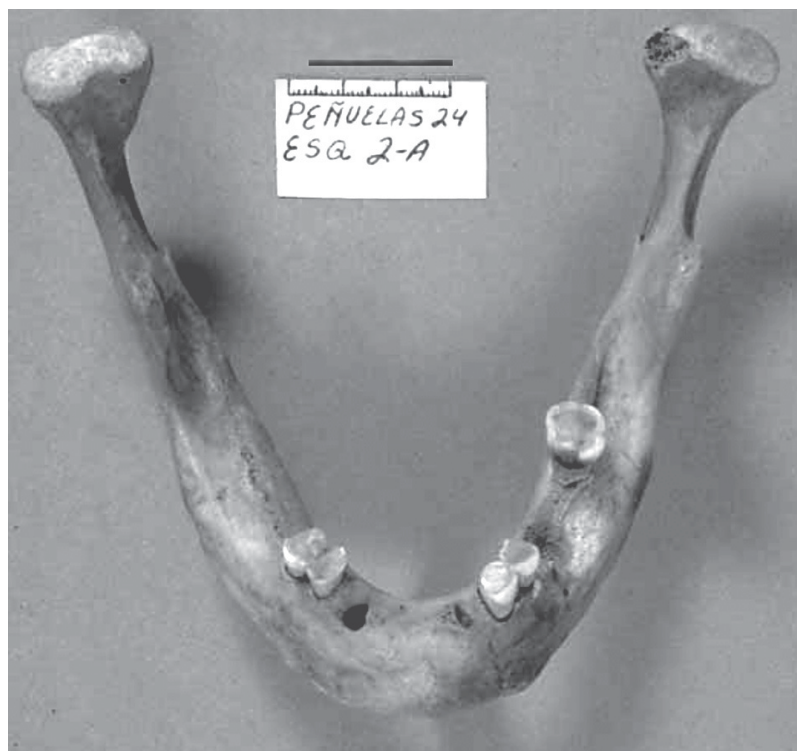

Fig. 12: ante mortem tooth loss, alveolus absorved; and dental wear (grades 7 and 8, cusp pattern obliterated, roots functioning at occlusal level). Bar: $3 \mathrm{~cm}$

TABLE IV

Frequency of paleopathologies; minimum number of individuals affected

\begin{tabular}{lc}
\hline Paleopathology & $\begin{array}{c}\text { Frequency of adults affected, } \\
\% \text { for the total sample }(\mathrm{n}=41)\end{array}$ \\
\hline Osteoarthritis vertebras & $12 / 29$ \\
Long bones & $12 / 29$ \\
Clavicle, sternum, ribs & $5 / 12$ \\
Sacrum, pelvis & $6 / 15$ \\
Foot and hand bones & $5 / 12$ \\
Sacrum, pelvis & $6 / 15$ \\
Trauma: long bones, cranium & $5 / 12$ \\
Periosteal reactions, osteomyelytis & $7 / 17$ \\
Cribra orbitalia & $5 / 12$ \\
Auditory exostoses & $2 / 5$
\end{tabular}

graphs with those of dry specimens.

Various forms of osteoarthritis: bony ankylosis of the seventh cervical and first thoracic, osteophyte formation and porosis of lumbars; and eburnation of left posterior, condylar region of femur can be seen in Figs 13, 14, 15. The osteoarthritis observed is age dependent- it is first observed in the beginning of the fourth decade.

Fig. 16 (radiograph), and Figs 17 and 18 indicate osteoarthritis of the pubic symphysis. Figs 19 and 20 show the sacrum and pelvis of a female in her sixth decade affected by fusion of the 5th lumbar and 1st sacral vertebras, with calcified DISH aging (Diffuse idiopathic skeletal hyperostosis - dense calcification/ossification anterior to the vertebral bodies).

In Figs 21, 22, and 23 there are the bones of a male in his sixth decade with trauma to the cranium (depressed fracture at lambda) and the mandible with loss of teeth antemortem. Figs 24 (radiograph), 25, and 26 indicate a proximal fracture of the left femur. Note the extent of 


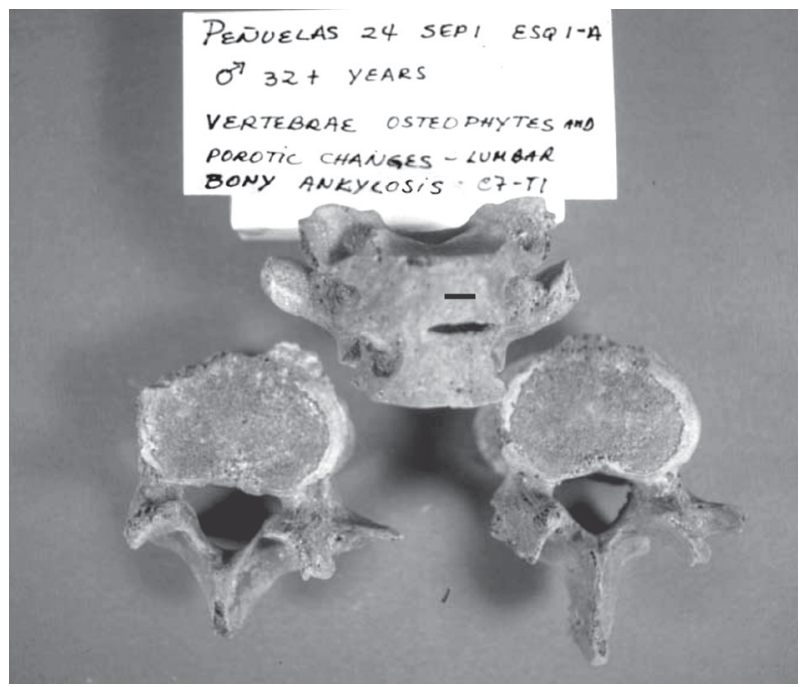

Fig. 13: bony ankylosis of seventh cervical and first thoracic vertebras. Bar: $1 \mathrm{~cm}$.

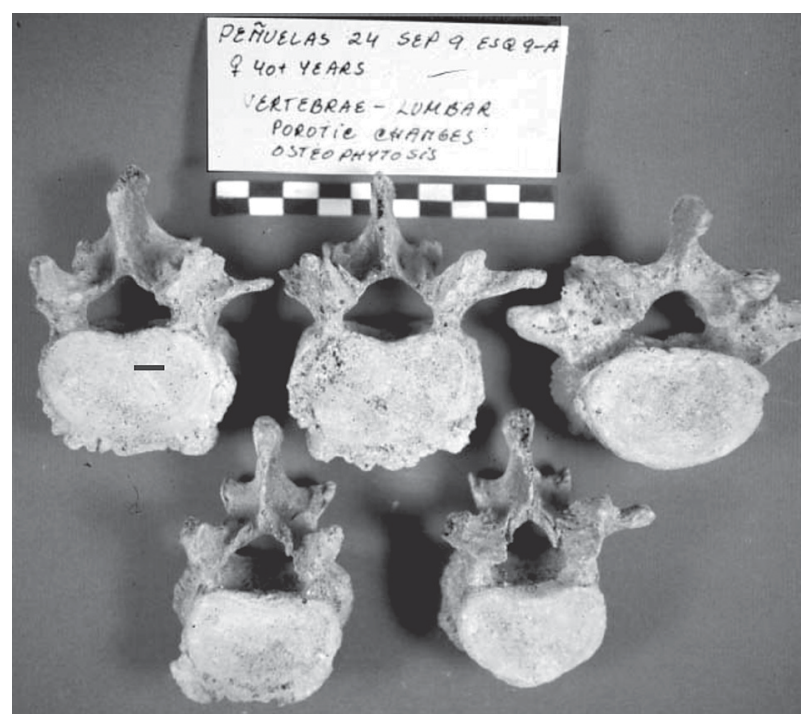

Fig. 14: lumbar osteophytes. Bar: $1 \mathrm{~cm}$.

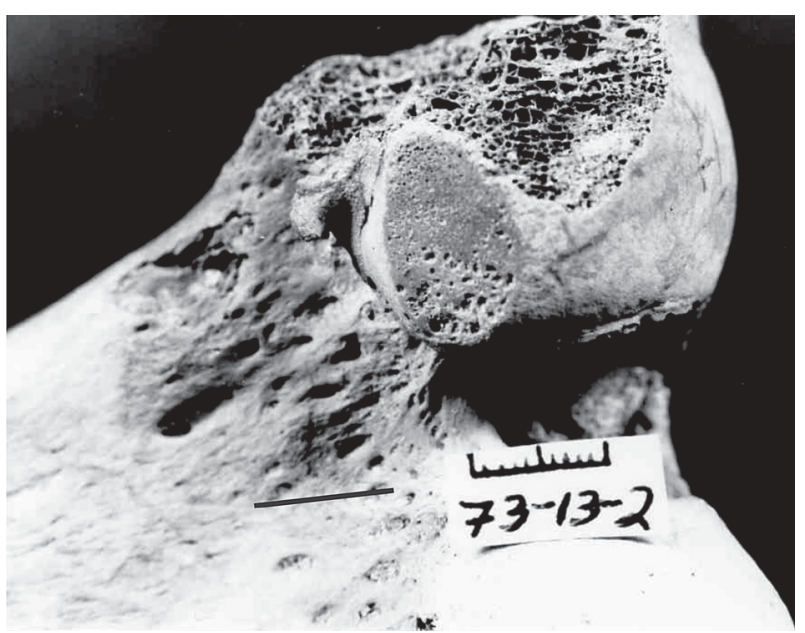

Fig. 15: eburnation of femoral condyle. Bar: $2 \mathrm{~cm}$.

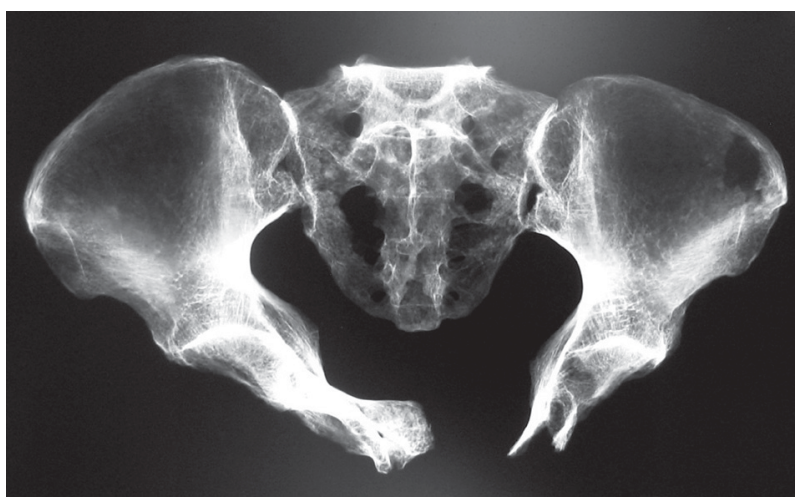

Fig. 16: osteoarthritis of the pubic symphysis.

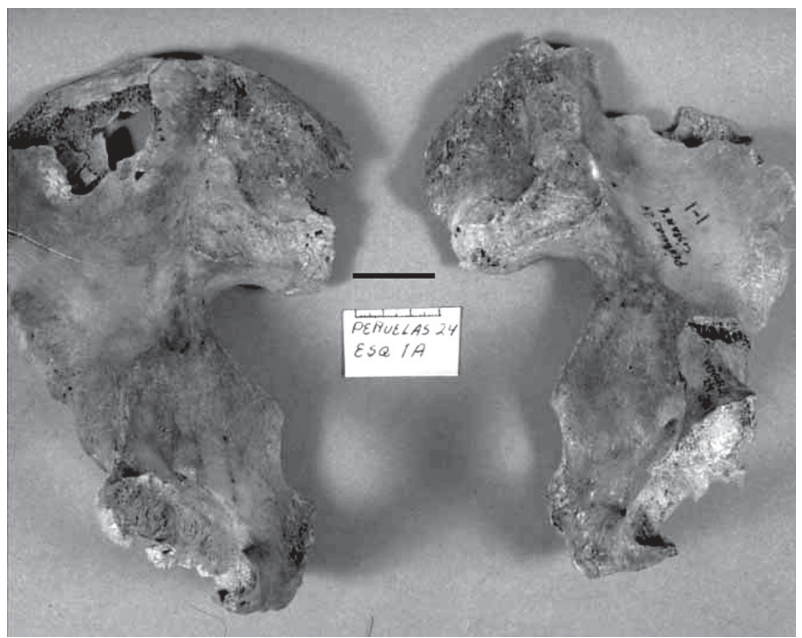

Fig. 17: osteoarthritis of the pubic symphysis. Bar: $3 \mathrm{~cm}$.

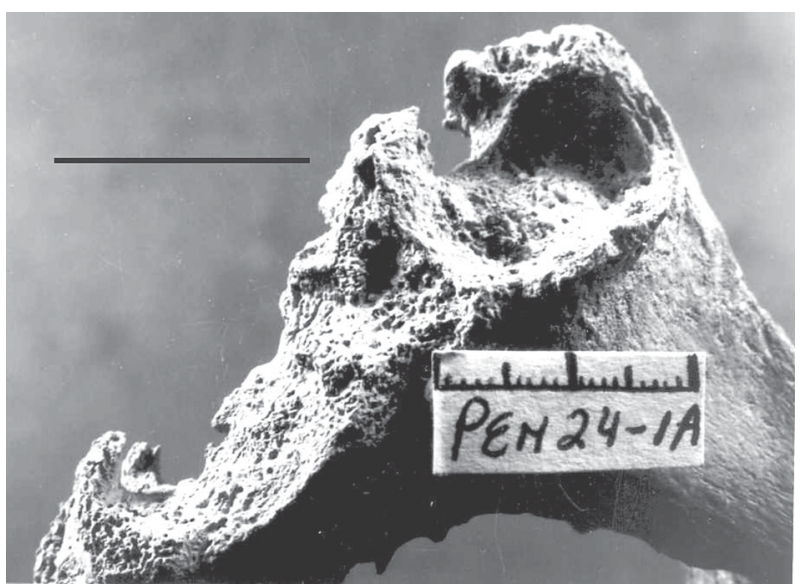

Fig. 18: osteoarthritis of the pubic symphysis (dorsal demiface and ventral rampart). Bar: $2 \mathrm{~cm}$.

the callus in the radiograph and photo (the lesser trochanter is no longer visible). The fracture shortened the femur of this female in her sixth decade by $1.5 \mathrm{~cm}$.

The radiograph of a right thoracic rib of a male in his third decade displaying a perforation inside a benign tumor, post traumatic cyst, or chronic abscess are shown in Figs 27 and 28. Fig. 29 shows the left orbit with cribra orbitalia of an adult female in her third decade. Fig. 30 is 


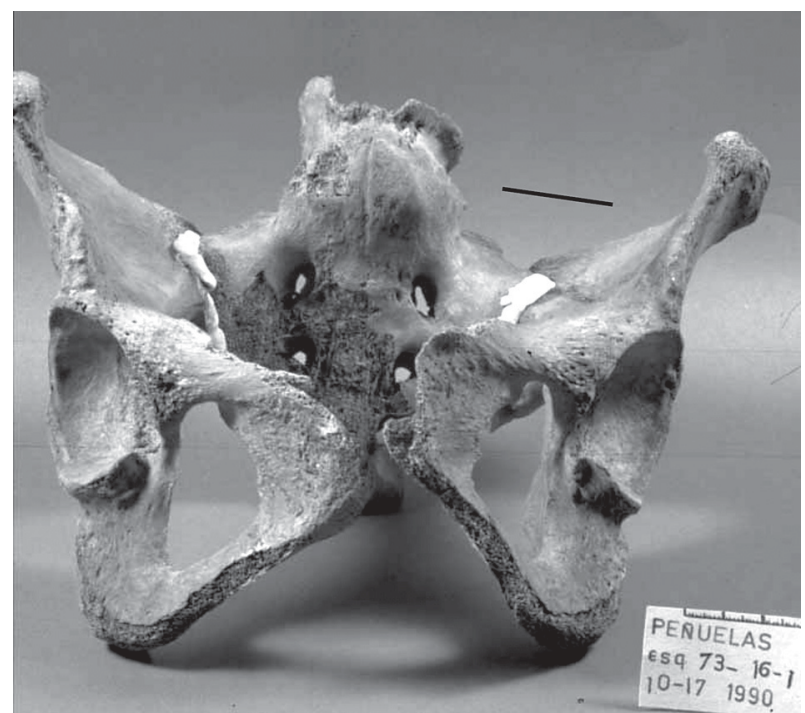

Fig. 19: fusion of the 5th lumbar and 1st sacral vertebra, with calcified DISH. Bar: $3 \mathrm{~cm}$.

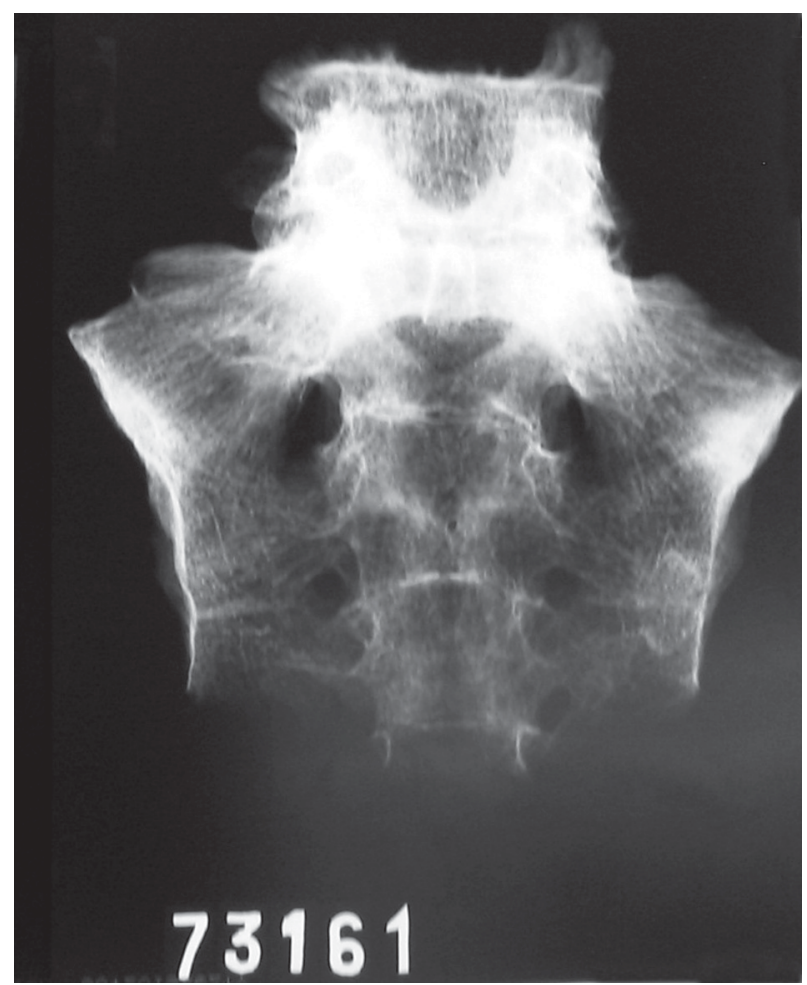

Fig. 20: radiograph of fusion of the 5th lumbar and 1 st sacral vertebra.

that of a male in his third decade with auditory exostoses of the right auditory meatus. The exostoses have restricted the meatus.

The long bones, in Figs 31 and 32, represent two adults in their third decade with periosteal reaction and osteomyelitis, of the tibial and femoral shafts, respectively. Figs 33 (radiograph) and 34 are those a female in her third decade. She has on the right femur osteoarthritis of the knee joint, a healed fracture, and a cyst. The radiograph (Fig.

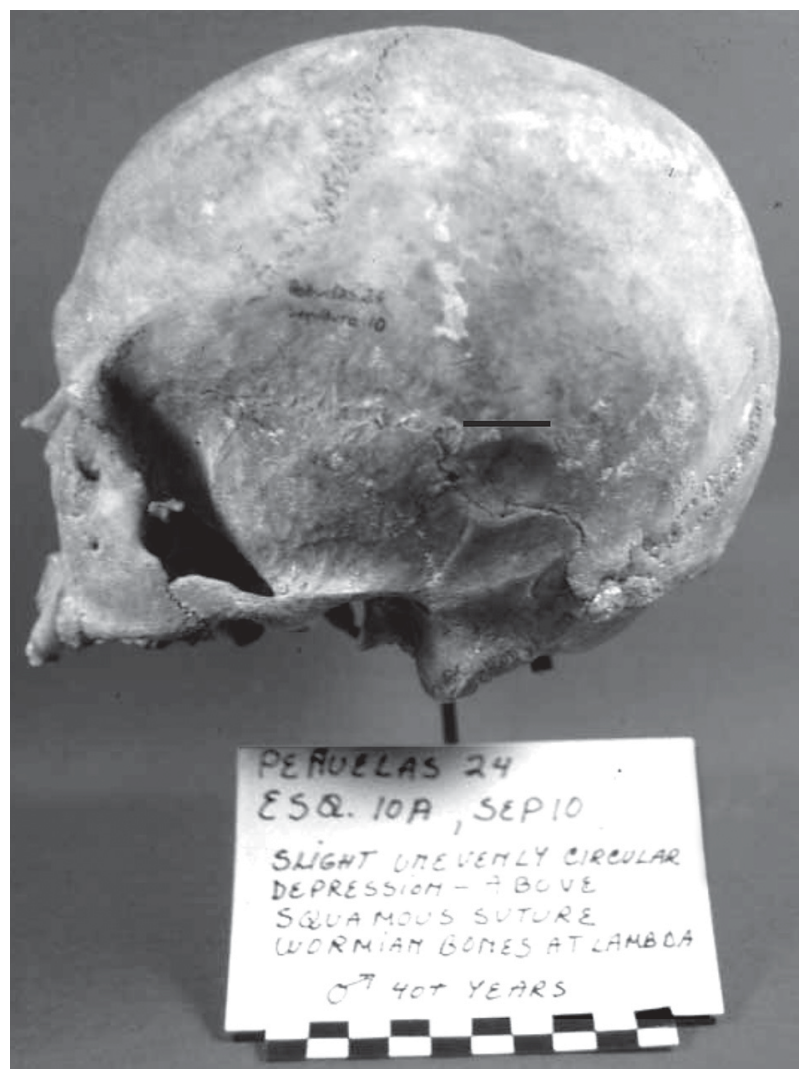

Fig. 21: depressed fracture at lambda. Bar: $2 \mathrm{~cm}$.

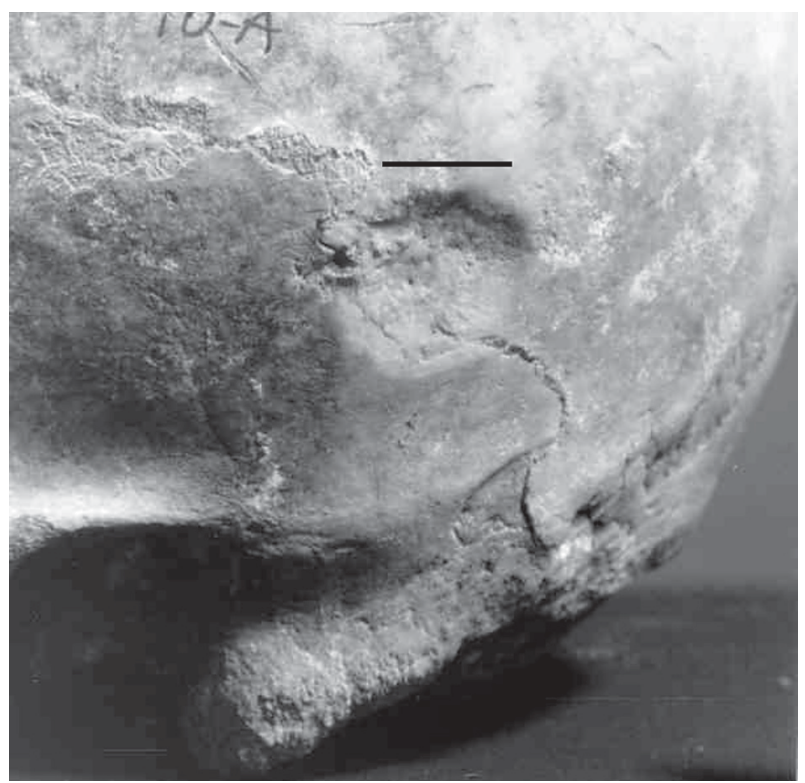

Fig. 22: depressed fracture at lambda. Bar: $2 \mathrm{~cm}$.

35) shows the femora and tibiae of a child 6-8 years of age. The bones diplay Harris Lines, or transverse radiopaque lines- indication of stress during childhood.

The Peñuelas sample also offered the opportunity to determine the type and frequency of artificial cranial deformation (ACD). The types of ACD provided in Neumann (1942) were used to identify those in the Peñuelas 


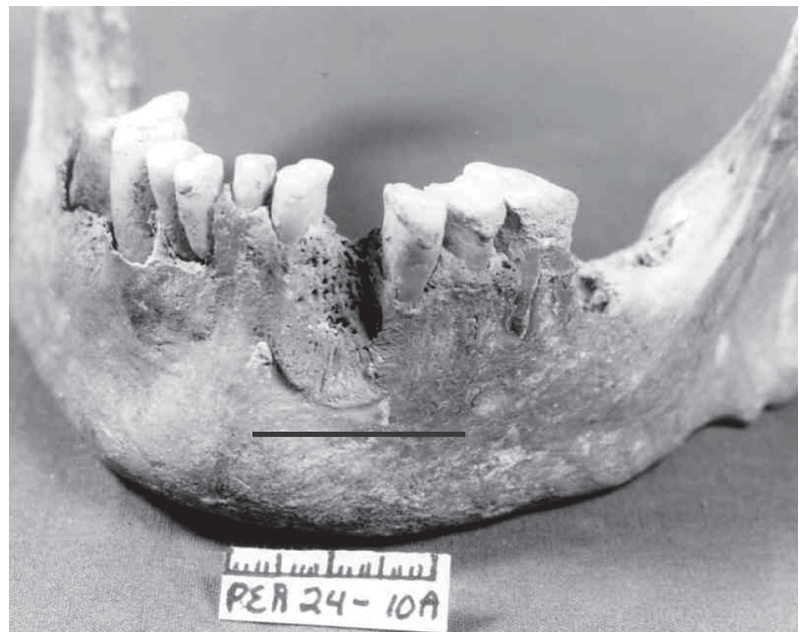

Fig. 23: ante mortem dental loss. Bar: $2 \mathrm{~cm}$.

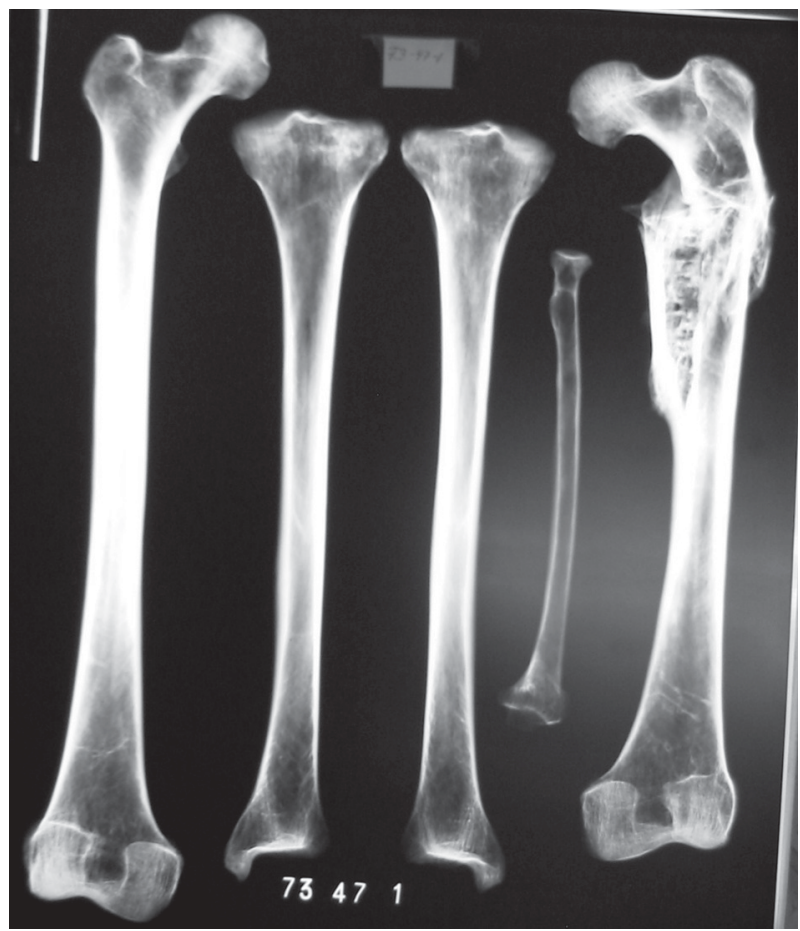

Fig. 24: radiograph of a proximal fracture of the left femur.

sample. There are 32 complete adult crania in this sample. Of these 7 females and 5 males, or $37.5 \%$ of the sample (males and females combined), display ACD of the tabular erect type with the characteristic frontooccipital flattening and lateral parietal bulges. Figs 36 and 37 display the sagittal views and superior view, respectively, of an adult male's skull affected by intentional cranial alteration. Note on the superior view the parietal bulges.

\section{DISCUSSION}

In the Peñuella sample there is moderate to severe degree of dental wear. This is very likely related to consumptions of gritty marine foods (shellfish) and from

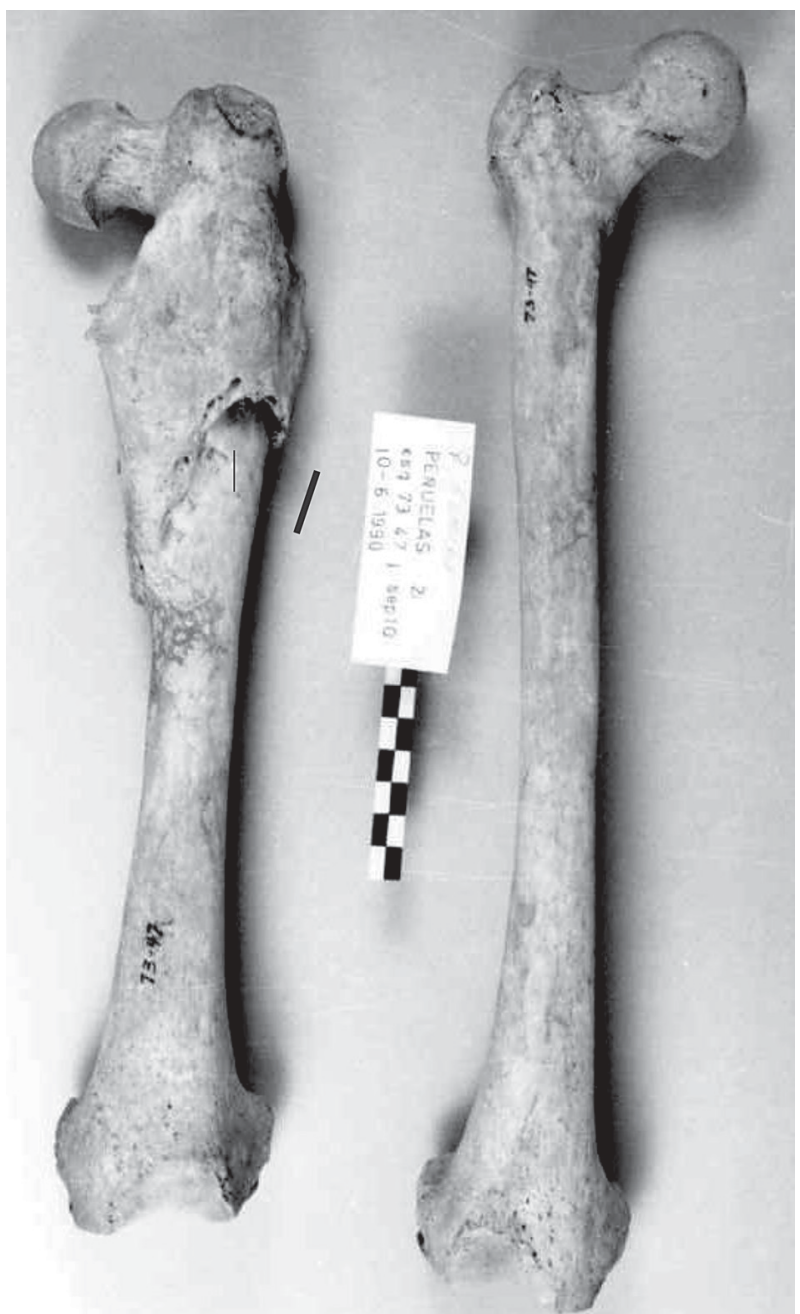

Fig. 25: proximal fracture of the left femur, anterior view. Bar: $2 \mathrm{~cm}$.

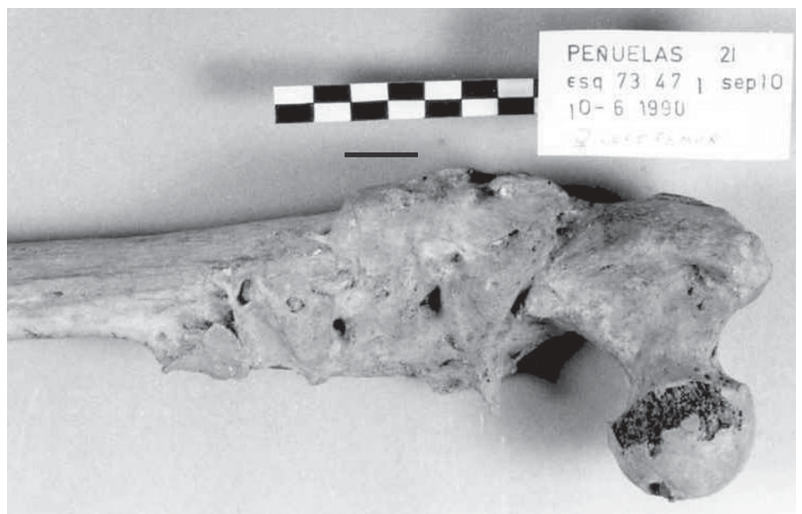

Fig. 26: proximal fracture of the left femur, posterior view. Bar: $2 \mathrm{~cm}$.

grittiness introduced from the grinding of vegetable foods. Mostly adults are affected and they display uneven wear. There are no sexual differences in frequencies of wear grade and the exposed dentine promoted abscess formation. 


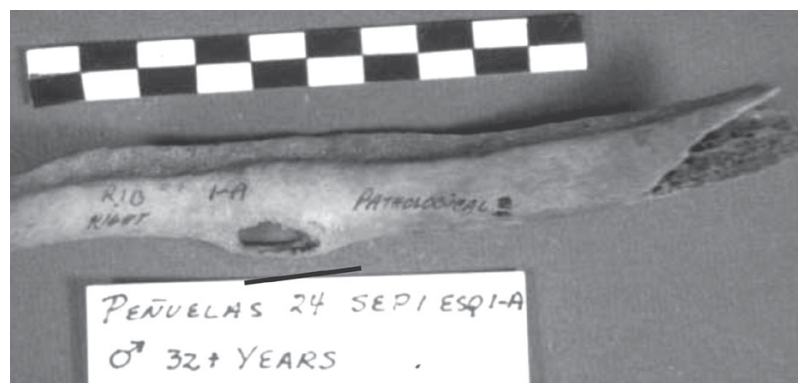

Fig. 27: right thoracic rib with a perforation inside a tumor, cyst, or abscess. Bar: $2 \mathrm{~cm}$.

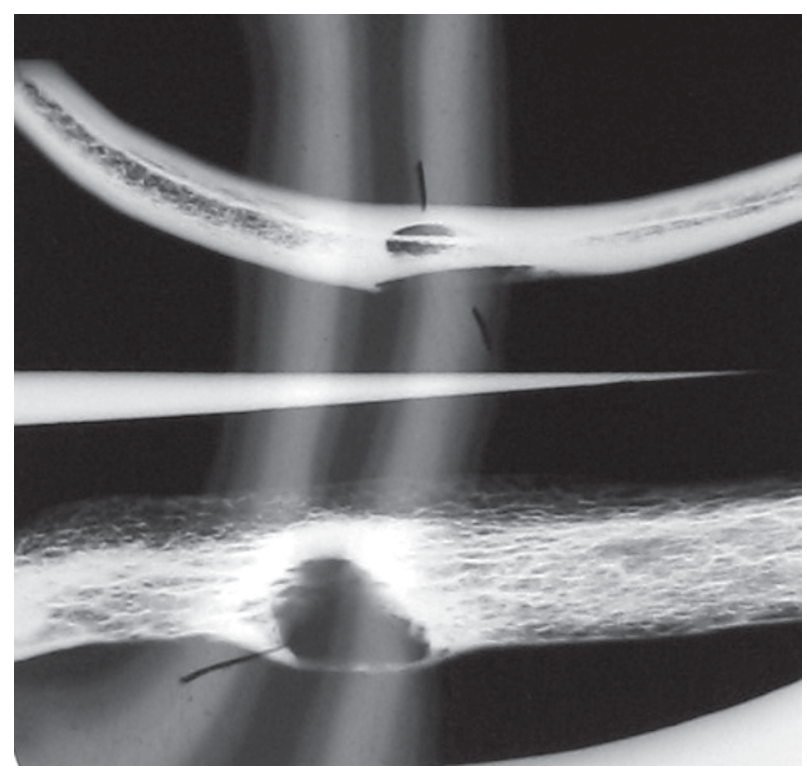

Fig. 28: radiograph of right thoracic rib.

The high frequencies of dental pathologies are very likely related to carbohydrates in the diet. The Diaguita were farmers who cultivated maize, squashes, and legumes (Ampuero 1989). There were no sexual differences observed in the frequency of dental paleopathologies.

In regards to the cranial and postcranial paleopathologies observed, osteoarthritis has the highest frequency, it is age related, and the lumbar vertebras are the most affected. The trauma observed is found on crania and long bones with only adults affected. This is very likely related to the movement in rugged terrain and a maritime subsistence practice where people collected shellfish in tidal pools of a rocky littoral that promoted bone injuries due to falls. No sexual differences in frequencies of skeletal paleopathologies were observed. There is a low frequency of "occupational" paleopathologies such as auditory exostoses observed, and both males and females practiced ACD of the tabular erect type.

We are interested in what the paleopathologies say about cultural and biological adaptations among prehistoric populations of Chile's semiarid littoral. This will help us to better understand the impact on health from the adoption of farming and a pastoral subsistence observed

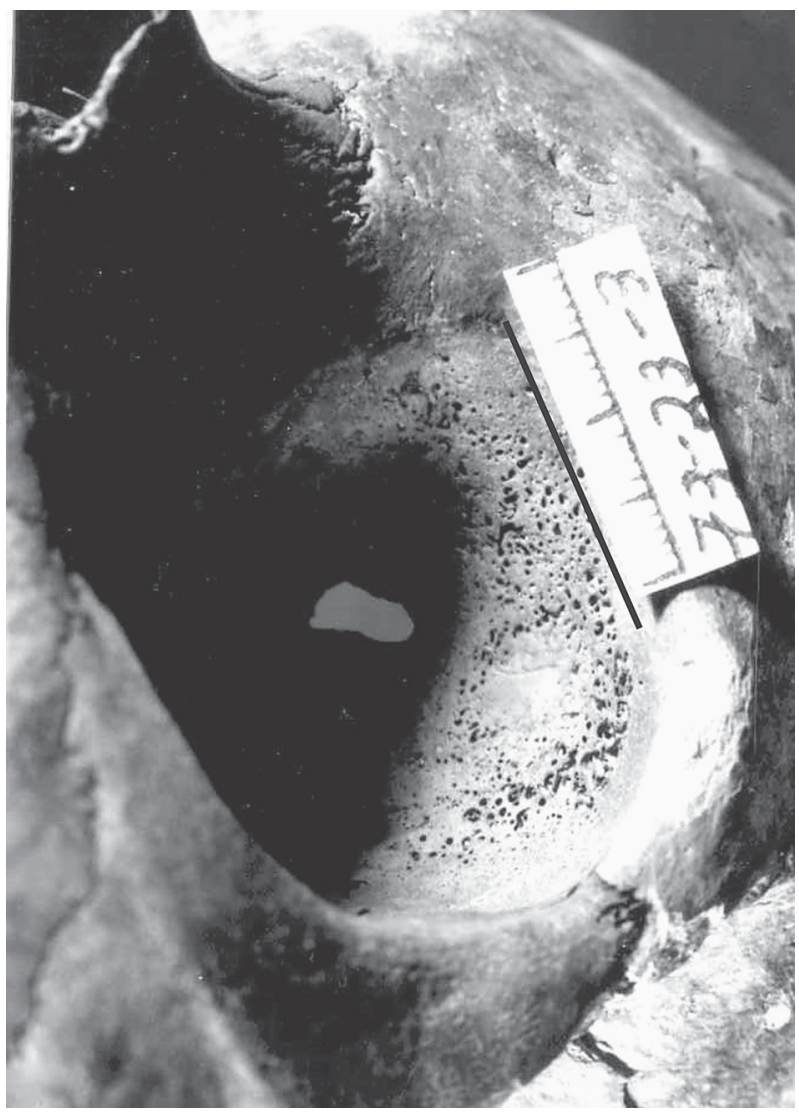

Fig 29: cribra orbitalia. Bar: $2 \mathrm{~cm}$.

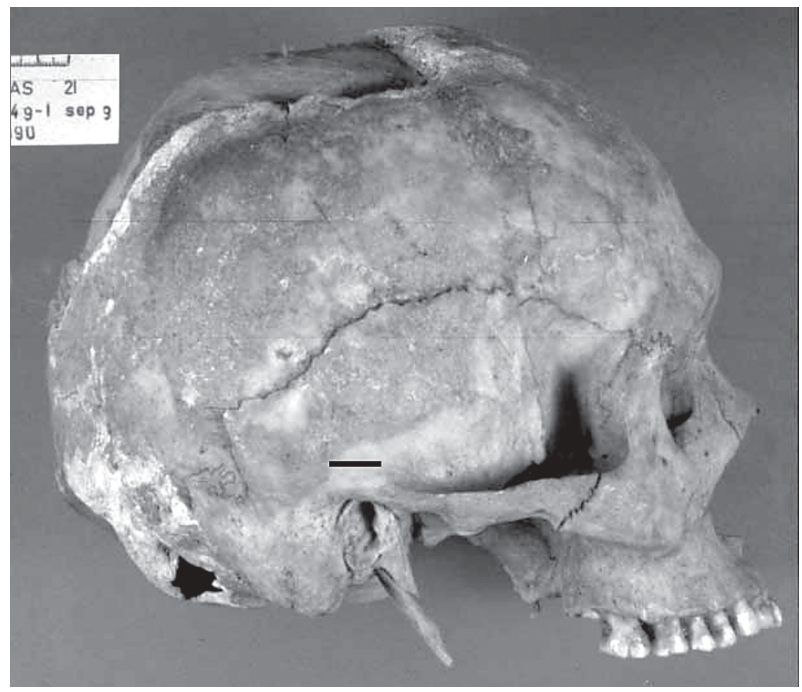

Fig. 30: auditory exostoses. Bar: $2 \mathrm{~cm}$.

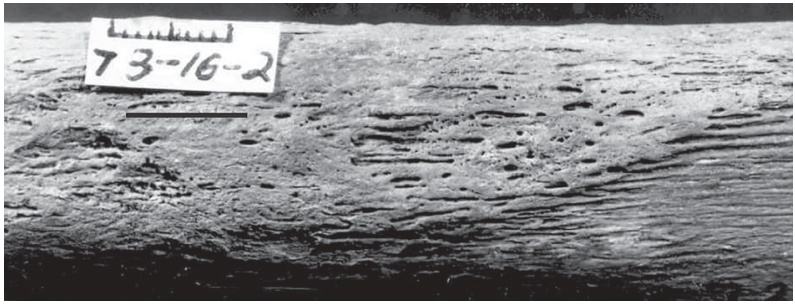

Fig. 31: periosteal reaction of the tibia (lateral view). Bar: $2 \mathrm{~cm}$. 


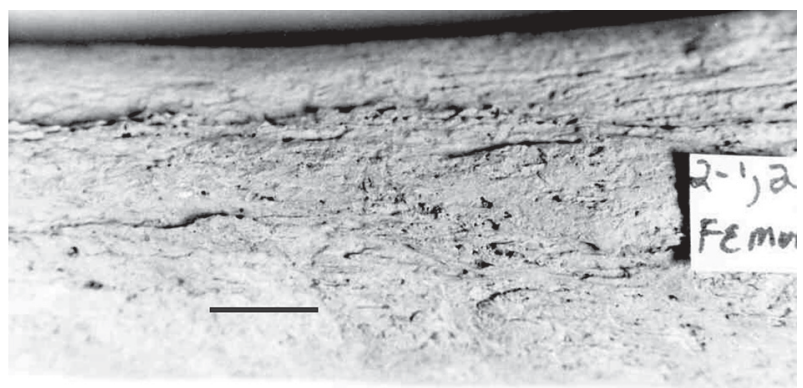

Fig. 32: osteomyelitis of the femur (anterior view). Bar: $2 \mathrm{~cm}$.

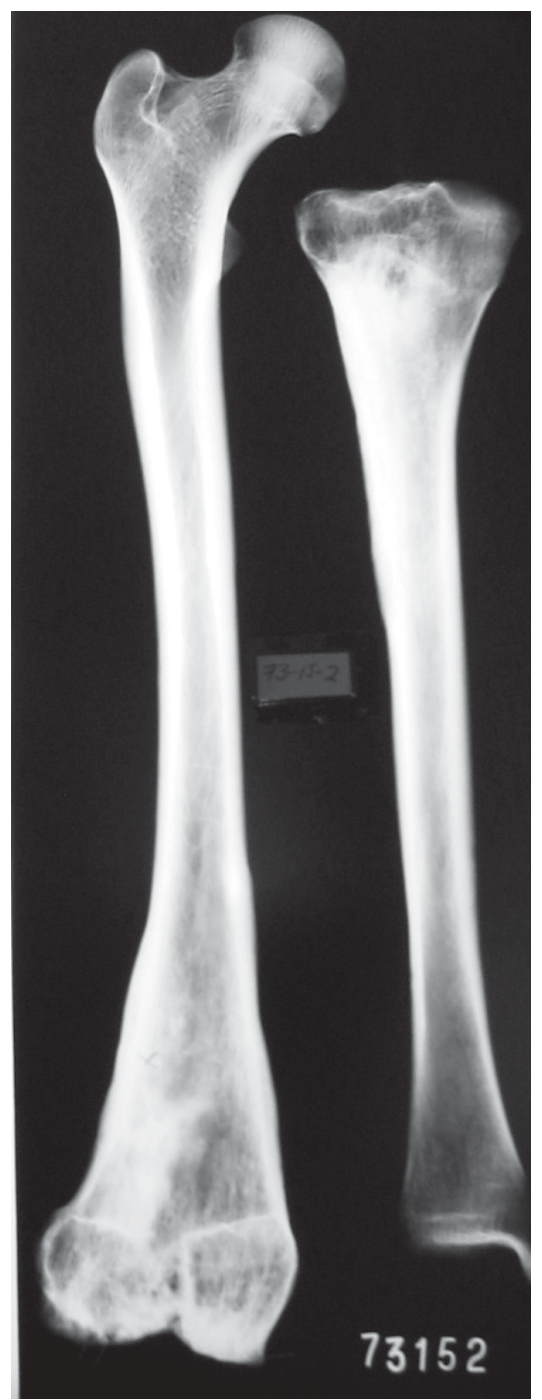

Fig. 33: radiograph of osteoarthritis of the knee joint with a healed fracture.

in the formative period of this region starting at around 1500 years ago. With the information on preservation status we can continue to develop better conservation measures to protect and conserve the skeletal remains for future and on-going bio-archaeological analyses. The next step in the bioarchaeology analyses is to document the paleopathologies at the microscopic level (see Fig. 38).

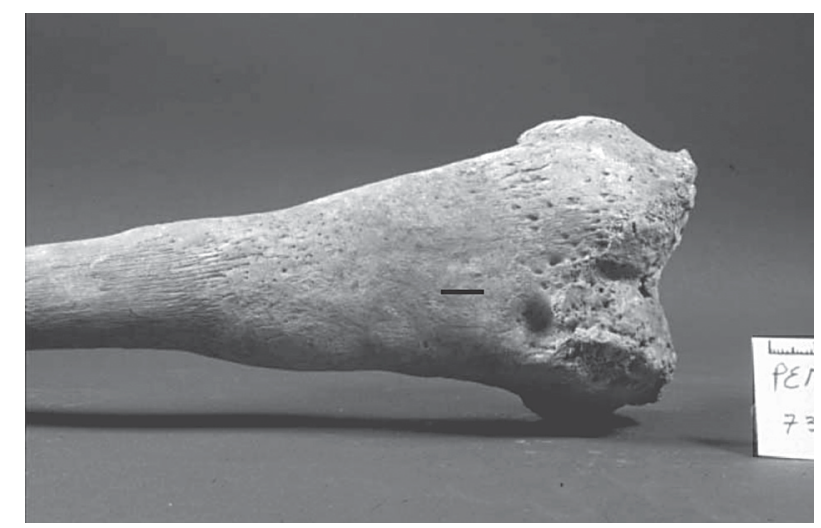

Fig. 34: osteoarthritis of the knee joint with a healed fracture, and cyst. Bar: $1 \mathrm{~cm}$.

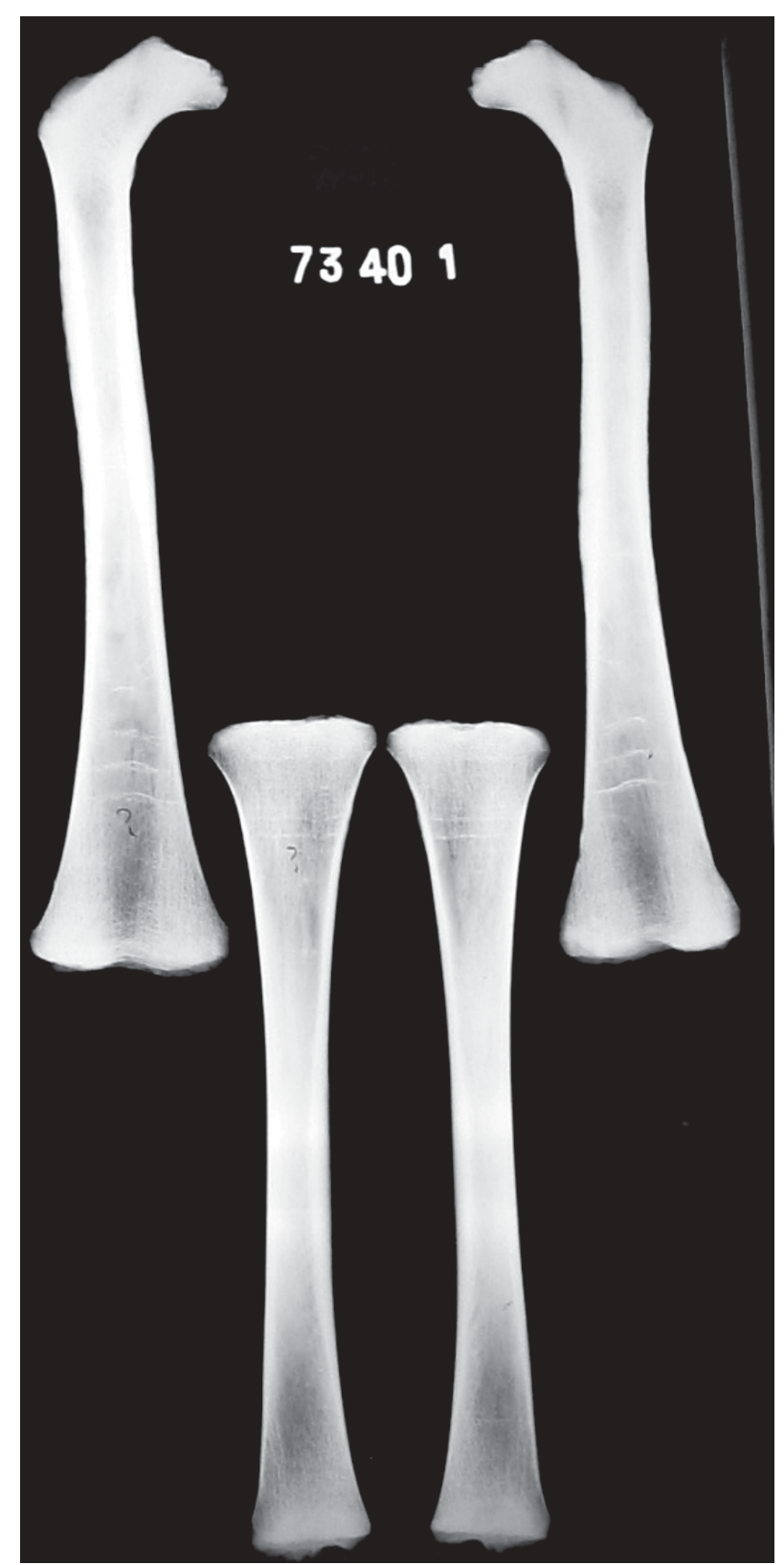

Fig. 35: Harris lines. 


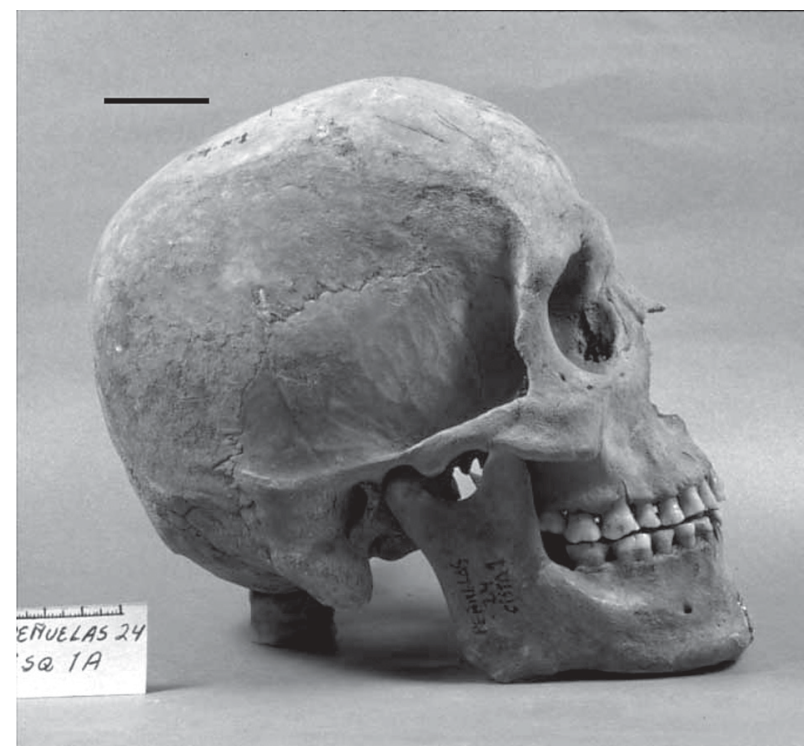

Fig. 36: sagittal view of artificial cranial deformation. Note the flattening of the occipital portion of the cranium. Bar: $3 \mathrm{~cm}$.

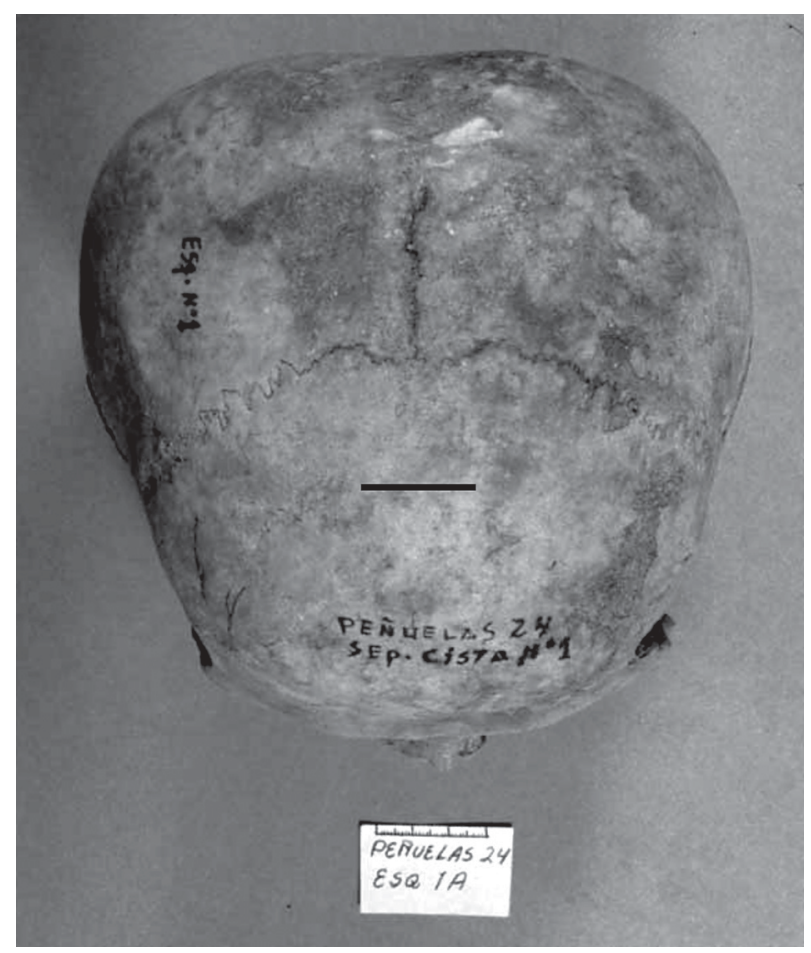

Fig. 37: superior view of artificial cranial deformation. Note the parietal bulges. Bar: $3 \mathrm{~cm}$.

This not only will help us to better understand how disease affects bone tissue but also to better diagnose disease.

\section{ACKNOWLEDGEMENTS}

To the Museo Arqueologico de La Serena, La Serena, Chile for their professional support and to Dr Morrie Kricun, of the University of Pennsylvania, for providing the radiograph diagnoses for the bone pathologies.

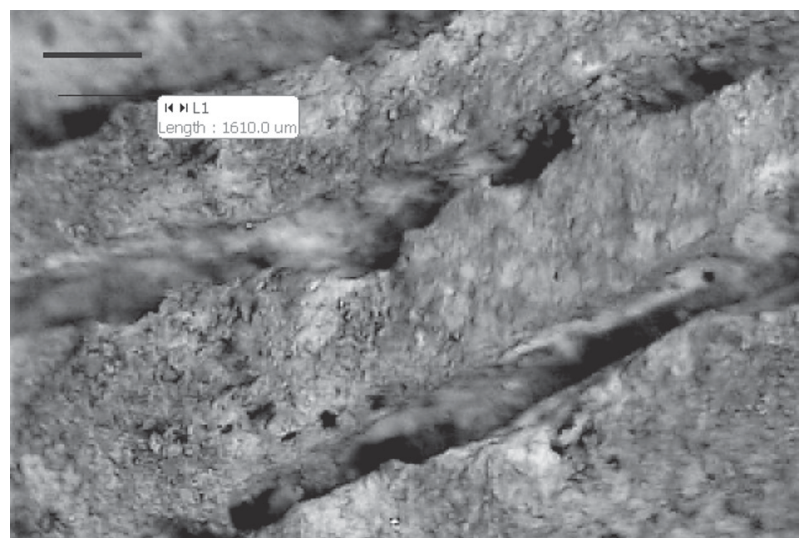

Fig 38: ten times magnification of periosteal reaction of the tibia (medial surface). Bar: $1610.0 \mu \mathrm{m}(1.6 \mathrm{~mm})$.

\section{REFERENCES}

Ampuero G 1989. La cultura Diaguita chilena. In Hidalgo J, Schiappacasse V, Niemeyer H, Aldunate A, Solimano I (eds), Prehistoria, Editorial Andress Bello, Santiago, Chile, p. 277287.

Bass W 1995. Human Osteology, Special Publication No.4 of the Missouri Archaeological Society, Columbia, Missouri.

Biskupovic M 1982. Excavaciones arqueológicas en la Parcela No. 21 de Penuelas-Coquimbo, Chile. Actas IX Congr Arqueol Bol Mus Arqueol de La Serena 18: 240-248.

Biskupovic M 1999. Excavación arqueológico en la planta Pisco Control. El Limari y sus Valles. Rev Est Prov 1: 1-6.

Cohen MN, Armelagos GJ 1984. Paleopathology at the Origins of Agriculture, Academic Press, New York.

Mays S 2000. The Archaeology of Human Bone, Routledge, London, New York.

Neumann GK 1942. Types of artificial cranial deformation in the Eastern United States. Am Antiquity 7: 306-310.

Ortner DJ, Putschar GJ 1985. Identification of Pathological Conditions in Human Skeletal Remains, Smith Inst Press, Washington.

Roberts C, Manchester K 1999. The Archaeology of Disease. Cornell University Press, Ithaca, New York.

Rosado MA1994. Health and Diet of Two Prehistoric Populations from Chile's Semiarid North, PhD Thesis, Department of Anthropology, Rutgers University, New Brunswick, New Jersey.

Rosado MA 1998. Dental health and diet of two prehistoric populations from Chile's semiarid North. Dental Anthropol 13:2-8.

Schmucker BJ 1985. Dental attrition: a correlative study of dietary and subsistence patterns in California and New Mexico Indians. In CF Merbs, RJ Miller (eds), Health and Disease in the Prehistoric Southwest, Arizona State Univesity Archaeological University Paper No. 34.

Swedlund AC, Armelagos GJ 1990. Disease in Populations in Transition, Bergin and Garbey, New York.

Steele D G, Bramblett C A 1988. The Anatomy and Biology of the Human Skeleton, Texas A \& M University Press, College Station, TX. 
